\title{
Effect of Crushed Eruca sativa Seeds Supplementation to Quail Ration on Lipid Profile Before and After Sexual Maturity
}

\author{
Abdullah F. Abdul-Majeed Sarmad Hashem Taha* \\ Department of Animal Production / College of Agriculture and Forestry/ \\ University of Mosul \\ Email : abdullahfathi@yahoo.com \\ sarmed.hashem89@gmail.com
}

\begin{abstract}
The study aimed to evaluate the effect of crushed Rocket salad (Eruca sativa) seeds on serum lipid profile and risk index of males and females quail before and after sexual maturity. A total of two hundred forty unsexed quail (Coturnix coturnix) (7 days aged) were randomly distributed into 4 groups (60 birds/ group, 5 replicates, 12 birds / replicate), the feed and water were allowed ad libitum, and the treatment continued till 77 days age, as follows:-

$1^{\text {st }}$ group(control): birds were reared on standard ration (without Eruca sativa seeds). $2^{\text {nd }}$ group: birds were reared on standard ration supplemented with $6 \mathrm{~g} / \mathrm{kg}$ crushed

Eruca sativa seeds from the age of 7 days till 42 days age.

$3^{\text {rd }}$ group: birds were reared on standard ration supplemented with $6 \mathrm{~g} / \mathrm{kg}$ crushed Eruca sativa seeds from the age of 42 days till 77 days age.

$4^{\text {th }}$ group: birds were reared on standard ration supplemented with $6 \mathrm{~g} / \mathrm{kg}$ crushed Eruca sativa seeds for the whole period of experiment (7 - 77 days).

The addition of crushed Eruca sativa seed don't change the level of blood glucose, but it improves serum lipid profile in males and females quail, especially when given in the early stage of growth before sexual maturity (Eruca sativa from 7 days age), and there was a significant decrease $(\mathrm{P} \leq 0.05)$ in the level of cholesterol, triglycerides and VLDL-C compared with control.

Also the addition of Eruca sativa seeds in the ration enhanced and significantly increased $(\mathrm{P} \leq 0.05)$ the level of HDL-C in males and females quail before sexual maturity ( $2^{\text {nd }}$ and $4^{\text {th }}$ groups), and there is no significant changes in the level of LDL-C compared with control, which reflected in improvement of risk index (LDL/HDL) especially when Eruca sativa seeds were added from 7 days age.

On the other hand, the addition of Eruca sativa seeds reduced the stress effects in males and females quail as represented by a significant decrease $(P \leq 0.05)$ in AST and ALT values as compared with the control group.

In conclusion, the addition of crushed Eruca sativa seeds improve the lipid profile and risk index, also reduce stress condition in males and females quail, especially when given in the growth stage and before sexual maturity.
\end{abstract}

Keyword: Eruca sativa, Rocket Salad, LDL, HDL, Quail.

\section{Received: 27/8/ 2018, Accepted: 7/11/2018}

\section{Introduction}

Rocket salad "Eruca sativa" is one of the most commonly used plants in many countries of world, including the Mediterranean region, particularly in Iraq, the Levant and Egypt (Uğur et al., 2010 ; Al-Eneezy, 2004), as it is of great importance for human and animal health as well as its various medicinal therapeutic properties,

* Part of M Sc. Dissertation of the second author 
according to the popular adage is used to increase sexual activity for both genders "Aphrodisiac", as it helps in increasing fertility and the production of sperm (Ansari and Ganaie, 2014).

Rocket salad or so-called "Arugula" it is used as leaves, seeds, extract or powder (Mossa et al., 1987). Arugula seeds and its leaves possess antioxidant activity(AbdulJalil, 2016), anti-lipid peroxidation (Abdel-Rahman et al., 2015), antioxidant vitamins and most of the vitamin B (Badeeet al., 2003 ; Carr et al., 2004 ; Barillari et al., 2005).

Eruca sativa seeds contain a wide range of nutritional elements and in different proportions depending on the environment in which the plant grows (Pignone and Ngu, 1995). The seeds are characterized by volatile oils (Flanders and Abdulkarim, 1985 ; El-Gengaihi et al., 2004) and many nutrient elements, proteins, vitamins (A and $\mathrm{C}$ ), carotenoids, mineral salts as well as containing glucosinolates (Bell and Wagstaff, 2014) and flavonoids vehicles (Barillari et al., 2005).

While the seeds oil mainly consists of fatty acids (Palmitic, Oleic, Linoleic, Linolenic and Erucic acids). The Eruca sativa seeds oil decrease serum cholesterol, triglycerides, LDL-C, VLDL-C and elevate the HDL-C level (El-Gengaihi et al., 2004; Mashi, 2017).

\section{Materials and Methods}

The study was conducted on a poultry farm of the Animal Production Department at the College of Agriculture and Forestry/University of Mosul. Two hundred forty unsexed males and females local quail (Coturnix Coturnix) were randomly distributed into four groups (60 birds/ group, 5 replicates, 12 birds/ replicate), the birds were reared in cages with dimensions $(50 \times 50 \times 50 \mathrm{~cm})$, the feed and water were allowed ad libitum throughout the study, and with appropriate requirements of lighting, ventilation and temperature depending on age of birds. The treatment continued till the age 77 days as follows:

$1^{\text {st }}$ group (control): birds were reared on standard ration (without Eruca sativa seeds).

$2^{\text {nd }}$ group: birds were reared on standard ration supplemented with $6 \mathrm{~g} / \mathrm{kg}$ crushed Eruca sativa seeds from the age of 7 days till 42 days age.

$3^{\text {rd }}$ group: birds were reared on standard ration supplemented with $6 \mathrm{~g} / \mathrm{kg}$ crushed Eruca sativa seeds from the age of 42 days till 77 days age.

$4^{\text {th }}$ group: birds were reared on standard ration supplemented with $6 \mathrm{~g} / \mathrm{kg}$ crushed Eruca sativa seeds for the whole period of experiment ( 7 - 77 days).

Eruca sativa seeds are bought from the local market, it crushed and mixed manually with ration before it was presented to the quail. The ration was formulated according to the standards of the National Research Council (Anonymous, 1994) which included: a starter ration of up to 35 days and a crude protein ratio of $22.4 \%$ and energy $2922.3 \mathrm{kcal} / \mathrm{kg}$, and then replaced with a finisher ration until the age of 77 days, crude protein was $21.5 \%$ and energy $3013.3 \mathrm{~kg} / \mathrm{kg}$.

Five birds from each group were slaughtered at age 42 and 77 days, blood collected in tubes without anticoagulants and the serum isolated then preserved at ($20^{\circ} \mathrm{c}$ ) until the biochemical tests were carried out, which included: estimation of the concentration of serum glucose, cholesterol, triglycerides, HDL-C, LDL-C, VLDL-C, risk index (LDL/HDL), AST, and ALT using Biosystems kits. 
The experiment was designed as C.R.D and the collected data were analyzed by one-way analysis of variance using statistical programs (S.A.S) (Anonymous, 2000). Then Duncan's Multiple Range test (Duncan, 1955) was used to determine the differences between means $(\mathrm{P} \leq 0.05)$, according to the Steel and Tories (1960).

\section{Results and Discussion}

Tables (1 and 2) showed that addition of crushed Eruca sativa to the ration had no effect on blood glucose. It may be due to that the level of blood glucose in birds is high, when compared with its level in mammals, and the preservation of blood glucose level is necessary as a source of energy for the brain and other body's cells (Al-Daraji et al., 2008), as well as its role in sustaining muscle movements, heart activity, nerve impulse transmission, and ions (Al-Dalaly, 1994).

Tables (1 and 2) showed that the addition of crushed Eruca sativa led to an improvement in the lipid profile of males and females quail, especially when added from Eruca sativa seeds was given early in the growth stage and before sexual maturity 7 days old. The levels of cholesterol and triglycerides in the growth stage and whole period were relatively good and balanced, especially in males.

Table (1) reveals that the cholesterol level in males was decreased significantly at age 42 days in $4^{\text {th }}$ group (Eruca sativa seeds from 7-77 days) as compared with control. Also, triglycerides were decreased significantly at 42 days age in $2^{\text {nd }}$ and $4^{\text {th }}$ groups, and at the age of 77 days in the $4^{\text {th }}$ group compared to control.

Table (2) showed a significantly decreased in triglycerides of females in all treatment groups at age 77 days as compared with control.

The addition of Eruca sativa to the ration before sexual maturity improves serum HDL-C in males significantly $(\mathrm{P} \leq 0.05)$ in the $2^{\text {nd }}$ and $4^{\text {th }}$ groups at the age 42 and 77 days, as compared with control group (Table 1), and in females, at the age 77 days in both $2^{\text {nd }}$ and $4^{\text {th }}$ groups, as compared with the control group at level $(\mathrm{P} \leq 0.05)($ Table 2$)$.

There were no significant differences $(\mathrm{P} \leq 0.05)$ among the birds of all groups in LDL-C level in males and females quail at the age 42 and 77 days, as compared with control group (Tables 1 and 2).

Very low-density lipoproteins (VLDL-C) decreased significantly in the serum of males $(\mathrm{P} \leq 0.05)$ in the $2^{\text {nd }}$ and $4^{\text {th }}$ groups at the age of 42 and 77 days as compared with control group (Table 1). In females, the VLDL-C was significantly decreased in all treatments at the age 77 days as compared with the control group at level $(\mathrm{P} \leq 0.05)$ (Table 2).

Tables ( 1 and 2 ) showed that the risk index values was improved significantly by the addition of Eruca sativa before sexual maturity ( 7 days) in both of the $2^{\text {nd }}$ and $4^{\text {th }}$ groups in the males of the quail at the age 42 , and at the age 77 days in females as compared with the control group at level $(\mathrm{P} \leq 0.05)$.

The results of the current study, research agreed with the findings of Razuki (2009) who used different levels of Eruca sativa (1, 2 and $3 \mathrm{~g} / \mathrm{kg}$ feed) in the chickens ration in regard to cholesterol level. 
Table (1): Effect of adding* crushed Eruca sativa seeds to the ration on blood glucose, serum total lipid profile and risk index in male quail at age 42 and 77 days.

\begin{tabular}{|c|c|c|c|c|c|c|c|c|c|c|c|c|c|c|}
\hline \multirow[b]{2}{*}{ Treatments } & \multicolumn{2}{|c|}{$\begin{array}{l}\text { Glucose } \\
\mathrm{mg} / \mathrm{dl}\end{array}$} & \multicolumn{2}{|c|}{$\begin{array}{c}\text { Cholesterol } \\
\mathrm{mg} / \mathrm{dl}\end{array}$} & \multicolumn{2}{|c|}{$\begin{array}{c}\text { Triglyceride } \\
\mathrm{mg} / \mathrm{dl}\end{array}$} & \multicolumn{2}{|c|}{$\begin{array}{c}\text { HDL-C } \\
\mathrm{mg} / \mathrm{dl}\end{array}$} & \multicolumn{2}{|c|}{$\begin{array}{c}\text { LDL-C } \\
\mathrm{mg} / \mathrm{dl}\end{array}$} & \multicolumn{2}{|c|}{$\begin{array}{l}\text { VLDL-C } \\
\mathrm{mg} / \mathrm{dl}\end{array}$} & \multicolumn{2}{|c|}{$\begin{array}{l}\text { Risk Index } \\
\text { LDL / HDL }\end{array}$} \\
\hline & $\begin{array}{c}42 \\
\text { days }\end{array}$ & $\begin{array}{r}77 \\
\text { days }\end{array}$ & $\begin{array}{c}42 \\
\text { days }\end{array}$ & $\begin{array}{r}77 \\
\text { days }\end{array}$ & $\begin{array}{c}42 \\
\text { days }\end{array}$ & $\begin{array}{r}77 \\
\text { days } \\
\end{array}$ & $\begin{array}{c}42 \\
\text { days }\end{array}$ & $\begin{array}{r}77 \\
\text { days } \\
\end{array}$ & $\begin{array}{c}42 \\
\text { days }\end{array}$ & $\begin{array}{r}77 \\
\text { days }\end{array}$ & $\begin{array}{c}42 \\
\text { days }\end{array}$ & $\begin{array}{c}77 \\
\text { days } \\
\end{array}$ & $\begin{array}{c}42 \\
\text { days }\end{array}$ & $\begin{array}{r}77 \\
\text { days }\end{array}$ \\
\hline $\begin{array}{c}1^{\text {st }} / \text { Control } \\
\text { (Without Eruca sativa } \\
\text { seeds) }\end{array}$ & $\begin{array}{c}306.60 \\
\pm 12.56 \\
\mathrm{~A}\end{array}$ & $\begin{array}{c}330.60 \\
\pm 8.76 \\
\mathrm{~A}\end{array}$ & $\begin{array}{c}308.20 \\
\pm 11.64 \\
\mathrm{~A}\end{array}$ & $\begin{array}{c}312.00 \\
\pm 23.37 \\
\mathrm{~A}\end{array}$ & $\begin{array}{c}128.60 \\
\pm 10.41 \\
\mathrm{~A}\end{array}$ & $\begin{array}{c}128.00 \\
\pm 7.14 \\
\mathrm{~A}\end{array}$ & $\begin{array}{c}46.60 \\
\pm 1.50 \\
\text { B }\end{array}$ & $\begin{array}{c}46.40 \\
\pm 3.04 \\
\text { B }\end{array}$ & $\begin{array}{c}231.88 \\
\pm 8.69 \\
\mathrm{~A}\end{array}$ & $\begin{array}{c}240.00 \\
\pm 24.11 \\
\mathrm{~A}\end{array}$ & $\begin{array}{c}25.72 \\
\pm 2.08 \\
\text { A }\end{array}$ & $\begin{array}{c}25.60 \\
\pm 1.43 \\
\mathrm{~A}\end{array}$ & $\begin{array}{c}4.99 \\
\pm 0.18 \\
\mathrm{~A}\end{array}$ & $\begin{array}{c}5.37 \\
\pm 0.93 \\
\mathrm{~A}\end{array}$ \\
\hline $\begin{array}{l}2^{\text {nd }} / \text { Growth stage } \\
\text { (Eruca sativa } \text { seeds } \\
\text { from } 7-42 \text { days) }\end{array}$ & $\begin{array}{c}308.20 \\
\pm 18.87 \\
\mathrm{~A}\end{array}$ & $\begin{array}{c}320.00 \\
\pm 12.39 \\
\mathrm{~A}\end{array}$ & $\begin{array}{c}276.00 \\
\pm 18.87 \\
\text { AB }\end{array}$ & $\begin{array}{c}331.00 \\
\pm 16.72 \\
\mathrm{~A}\end{array}$ & $\begin{array}{c}94.60 \\
\pm 12.70 \\
\text { B }\end{array}$ & $\begin{array}{c}108.60 \\
\pm 6.85 \\
\mathrm{AB}\end{array}$ & $\begin{array}{c}52.80 \\
\pm 1.77 \\
\mathrm{~A}\end{array}$ & $\begin{array}{c}56.80 \\
\pm 1.36 \\
\mathrm{~A}\end{array}$ & $\begin{array}{c}204.28 \\
\pm 16.82 \\
\mathrm{~A}\end{array}$ & $\begin{array}{c}252.48 \\
\pm 17.30 \\
\mathrm{~A}\end{array}$ & $\begin{array}{c}18.92 \\
\pm 2.54 \\
\text { B }\end{array}$ & $\begin{array}{l}21.32 \\
\pm 1.32 \\
\text { B }\end{array}$ & $\begin{array}{c}3.86 \\
\pm 0.26 \\
\quad \mathrm{~B} \\
\end{array}$ & $\begin{array}{c}4.48 \\
\pm 0.41 \\
\mathrm{~A}\end{array}$ \\
\hline $\begin{array}{l}3^{\text {rd }} / \text { Production stage } \\
\text { (Eruca sativa seeds } \\
\text { from 42-77 days) }\end{array}$ & $\begin{array}{c}313.60 \\
\pm 1.69 \\
\mathrm{~A}\end{array}$ & $\begin{array}{c}323.20 \\
\pm 7.13 \\
\mathrm{~A}\end{array}$ & $\begin{array}{c}278.00 \\
\pm 13.74 \\
\text { AB }\end{array}$ & $\begin{array}{c}326.60 \\
\pm 6.13 \\
\mathrm{~A}\end{array}$ & $\begin{array}{c}108.60 \\
\pm 9.91 \\
\mathrm{AB}\end{array}$ & $\begin{array}{c}116.40 \\
\pm 5.73 \\
\mathrm{AB}\end{array}$ & $\begin{array}{c}50.00 \\
\pm 2.12 \\
\mathrm{AB}\end{array}$ & $\begin{array}{c}52.40 \\
\pm 2.91 \\
\mathrm{AB}\end{array}$ & $\begin{array}{c}206.28 \\
\pm 14.98 \\
\mathrm{~A}\end{array}$ & $\begin{array}{c}250.92 \\
\pm 6.13 \\
\mathrm{~A}\end{array}$ & $\begin{array}{c}21.72 \\
\pm 1.98 \\
\mathrm{AB}\end{array}$ & $\begin{array}{c}23.28 \\
\pm 1.15 \\
\mathrm{AB}\end{array}$ & $\begin{array}{c}4.19 \\
\pm 0.41 \\
\mathrm{AB}\end{array}$ & $\begin{array}{c}4.87 \\
\pm 0.37 \\
\mathrm{~A}\end{array}$ \\
\hline $\begin{array}{l}4^{\text {th }} / \text { Total stage } \\
\text { (Eruca sativa seeds } \\
\text { from } 7-77 \text { days) }\end{array}$ & $\begin{array}{c}329.00 \\
\pm 7.89 \\
\mathrm{~A}\end{array}$ & $\begin{array}{c}302.80 \\
\pm 12.10 \\
\mathrm{~A}\end{array}$ & $\begin{array}{c}260.00 \\
\pm 13.18 \\
\text { B }\end{array}$ & $\begin{array}{c}319.80 \\
\pm 14.80 \\
\mathrm{~A}\end{array}$ & $\begin{array}{l}89.20 \\
\pm 6.51 \\
\quad \text { B }\end{array}$ & $\begin{array}{c}96.60 \\
\pm 6.86 \\
\text { B }\end{array}$ & $\begin{array}{c}52.60 \\
\pm 1.69 \\
\mathrm{~A}\end{array}$ & $\begin{array}{c}57.60 \\
\pm 3.08 \\
\text { A }\end{array}$ & $\begin{array}{c}190.16 \\
\pm 12.35 \\
\mathrm{~A}\end{array}$ & $\begin{array}{c}242.88 \\
\pm 12.99 \\
\mathrm{~A}\end{array}$ & $\begin{array}{c}17.84 \\
\pm 1.30 \\
\text { B }\end{array}$ & $\begin{array}{c}19.32 \\
\pm 1.37 \\
\text { B }\end{array}$ & $\begin{array}{c}3.63 \\
\pm 0.24 \\
\text { B }\end{array}$ & $\begin{array}{c}4.23 \\
\pm 0.17 \\
\text { A }\end{array}$ \\
\hline
\end{tabular}

- Values are represent : Means \pm Standard Error.

- Different letters in each column mean significant differences at $(\mathrm{P} \leq 0.05)$.

-*Adding $6 \mathrm{~g} / \mathrm{kg}$ feed of crushed Eruca sativa seeds. 
Table (2): Effect of adding* crushed Eruca sativa seeds to the ration on blood glucose, serum total lipid profile and risk index in female quail at age 42 and 77 days.

\begin{tabular}{|c|c|c|c|c|c|c|c|c|c|c|c|c|c|c|}
\hline \multirow[b]{2}{*}{ Treatments } & \multicolumn{2}{|c|}{$\begin{array}{l}\text { Glucose } \\
\mathrm{mg} / \mathrm{dl}\end{array}$} & \multicolumn{2}{|c|}{$\begin{array}{l}\text { Cholesterol } \\
\mathrm{mg} / \mathrm{dl}\end{array}$} & \multicolumn{2}{|c|}{$\begin{array}{l}\text { Triglyceride } \\
\mathrm{mg} / \mathrm{dl}\end{array}$} & \multicolumn{2}{|c|}{$\begin{array}{c}\mathrm{HDL}-\mathrm{C} \\
\mathrm{mg} / \mathrm{dl}\end{array}$} & \multicolumn{2}{|c|}{$\begin{array}{c}\text { LDL-C } \\
\mathrm{mg} / \mathrm{dl}\end{array}$} & \multicolumn{2}{|c|}{$\begin{array}{l}\text { VLDL-C } \\
\mathrm{mg} / \mathrm{dl}\end{array}$} & \multicolumn{2}{|c|}{$\begin{array}{l}\text { Risk Index } \\
\text { LDL / HDL }\end{array}$} \\
\hline & $\begin{array}{c}42 \\
\text { days }\end{array}$ & $\begin{array}{c}77 \\
\text { days }\end{array}$ & $\begin{array}{c}42 \\
\text { days }\end{array}$ & $\begin{array}{r}77 \\
\text { days } \\
\end{array}$ & $\begin{array}{c}42 \\
\text { days }\end{array}$ & $\begin{array}{c}77 \\
\text { days }\end{array}$ & $\begin{array}{c}42 \\
\text { days }\end{array}$ & $\begin{array}{r}77 \\
\text { days } \\
\end{array}$ & $\begin{array}{c}42 \\
\text { days }\end{array}$ & $\begin{array}{c}77 \\
\text { days }\end{array}$ & $\begin{array}{c}42 \\
\text { days }\end{array}$ & $\begin{array}{c}77 \\
\text { days }\end{array}$ & $\begin{array}{c}42 \\
\text { days }\end{array}$ & $\begin{array}{r}77 \\
\text { days } \\
\end{array}$ \\
\hline $\begin{array}{c}1^{\text {st }} / \text { Control } \\
\text { (Without Eruca sativa } \\
\text { seeds) }\end{array}$ & $\begin{array}{c}222.80 \\
\pm 16.11 \\
\text { A }\end{array}$ & $\begin{array}{c}292.80 \\
\pm 10.46 \\
\text { A }\end{array}$ & $\begin{array}{c}289.20 \\
\pm 11.00 \\
\text { A }\end{array}$ & $\begin{array}{c}340.40 \\
\pm 19.27 \\
\text { A }\end{array}$ & $\begin{array}{c}714.60 \\
\pm 15.25 \\
\text { A }\end{array}$ & $\begin{array}{c}768.80 \\
\pm 79.58 \\
\text { A }\end{array}$ & $\begin{array}{c}20.00 \\
\pm 1.87 \\
\mathrm{~A}\end{array}$ & $\begin{array}{l}11.00 \\
\pm 0.71 \\
\mathrm{~B}\end{array}$ & $\begin{array}{c}126.20 \\
\pm 10.02 \\
\text { A }\end{array}$ & $\begin{array}{c}175.64 \\
\pm 33.54 \\
\mathrm{~A}\end{array}$ & $\begin{array}{c}143.00 \\
\pm 3.05 \\
\mathrm{~A}\end{array}$ & $\begin{array}{c}153.76 \\
\pm 15.92 \\
\mathrm{~A}\end{array}$ & $\begin{array}{c}6.50 \\
\pm 0.68 \\
\mathrm{~A}\end{array}$ & $\begin{array}{c}16.32 \\
\pm 3.47 \\
\text { A }\end{array}$ \\
\hline $\begin{array}{l}2^{\text {bd }} / \text { Growth stage } \\
\text { (Eruca sativa seeds } \\
\text { from } 7-42 \text { days) }\end{array}$ & $\begin{array}{c}247.00 \\
\pm 5.19 \\
\text { A }\end{array}$ & $\begin{array}{c}300.60 \\
\pm 7.12 \\
\mathrm{~A}\end{array}$ & $\begin{array}{c}320.40 \\
\pm 31.05 \\
\text { A }\end{array}$ & $\begin{array}{c}294.80 \\
\pm 22.14 \\
\text { A }\end{array}$ & $\begin{array}{c}744.20 \\
\pm 17.86 \\
\text { A }\end{array}$ & $\begin{array}{l}579.80 \\
\pm 19.21 \\
\quad \text { B }\end{array}$ & $\begin{array}{c}21.00 \\
\pm 1.48 \\
\mathrm{~A}\end{array}$ & $\begin{array}{r}18.60 \\
\pm 1.81 \\
\mathrm{~A}\end{array}$ & $\begin{array}{c}150.40 \\
\pm 32.68 \\
\mathrm{~A}\end{array}$ & $\begin{array}{c}160.24 \\
\pm 21.39 \\
\mathrm{~A}\end{array}$ & $\begin{array}{c}149.00 \\
\pm 3.63 \\
\mathrm{~A}\end{array}$ & $\begin{array}{c}115.96 \\
\pm 3.84 \\
\text { B }\end{array}$ & $\begin{array}{c}7.46 \\
\pm 1.62 \\
\mathrm{~A}\end{array}$ & $\begin{array}{c}8.95 \\
\pm 1.59 \\
\mathrm{~B}\end{array}$ \\
\hline $\begin{array}{l}3^{\text {rd }} / \text { Production stage } \\
\text { (Eruca sativa seeds } \\
\text { from } 42-77 \text { days) }\end{array}$ & $\begin{array}{c}217.80 \\
\pm 6.34 \\
\mathrm{~A}\end{array}$ & $\begin{array}{c}308.40 \\
\pm 9.64 \\
\mathrm{~A}\end{array}$ & $\begin{array}{c}284.20 \\
\pm 21.16 \\
\text { A }\end{array}$ & $\begin{array}{c}312.60 \\
\pm 22.94 \\
\text { A }\end{array}$ & $\begin{array}{c}717.80 \\
\pm 17.65 \\
\text { A }\end{array}$ & $\begin{array}{c}575.60 \\
\pm 17.08 \\
\quad \mathrm{~B}\end{array}$ & $\begin{array}{c}22.80 \\
\pm 0.97 \\
\mathrm{~A}\end{array}$ & $\begin{array}{c}14.60 \\
\pm 0.60 \\
\quad \mathrm{~B}\end{array}$ & $\begin{array}{c}117.60 \\
\pm 23.25 \\
\text { A }\end{array}$ & $\begin{array}{c}182.88 \\
\pm 24.24 \\
\mathrm{~A}\end{array}$ & $\begin{array}{c}143.80 \\
\pm 3.40 \\
\mathrm{~A}\end{array}$ & $\begin{array}{c}115.12 \\
\pm 3.42 \\
\text { B }\end{array}$ & $\begin{array}{c}5.32 \\
\pm 1.28 \\
\mathrm{~A}\end{array}$ & $\begin{array}{c}12.65 \\
\pm 1.87 \\
\mathrm{AB}\end{array}$ \\
\hline $\begin{array}{l}4^{\text {th }} / \text { Total stage } \\
\text { (Eruca sativa } \text { seeds } \\
\text { from } 7-77 \text { days) }\end{array}$ & $\begin{array}{c}229.20 \\
\pm 10.85 \\
\text { A }\end{array}$ & $\begin{array}{c}314.40 \\
\pm 11.62 \\
\text { A }\end{array}$ & $\begin{array}{c}333.80 \\
\pm 26.11 \\
\mathrm{~A}\end{array}$ & $\begin{array}{c}305.20 \\
\pm 25.59 \\
\text { A }\end{array}$ & $\begin{array}{c}750.20 \\
\pm 16.30 \\
\text { A }\end{array}$ & $\begin{array}{c}564.20 \\
\pm 9.61 \\
\text { B }\end{array}$ & $\begin{array}{c}22.00 \\
\pm 1.87 \\
\mathrm{~A}\end{array}$ & $\begin{array}{c}20.80 \\
\pm 1.39 \\
\mathrm{~A}\end{array}$ & $\begin{array}{c}161.80 \\
\pm 27.66 \\
\text { A }\end{array}$ & $\begin{array}{c}171.56 \\
\pm 26.11 \\
\mathrm{~A}\end{array}$ & $\begin{array}{c}150.00 \\
\pm 3.21 \\
\mathrm{~A}\end{array}$ & $\begin{array}{c}112.84 \\
\pm 1.92 \\
\text { B }\end{array}$ & $\begin{array}{c}7.89 \\
\pm 1.84 \\
\mathrm{~A}\end{array}$ & $\begin{array}{c}8.50 \\
\pm 1.55 \\
\text { B }\end{array}$ \\
\hline
\end{tabular}

- Values are represent : Means \pm Standard Error.

- Different letters in each column mean significant differences at $(\mathrm{P} \leq 0.05)$.

-*Adding $6 \mathrm{~g} / \mathrm{kg}$ feed of crushed Eruca sativa seeds. 
El-Gengaihi et al., (2004) also explained that the hypocholesterolemic effect of Eruca sativa oil may be due to its unsaturated fatty acids $(85 \%)$ or due to the effect of $\beta$-sitosterol (6.5\%) which reduces the cholesterol absorption from the intestine so it decreases its level in the blood.

The hypocholesterolemic effect of Eruca sativa may be due to the it's flavonoids compounds (Badee et al., 2003).

The Eruca sativa seed constituents as the vitamin $\mathrm{C}$ and carotenoids they may have a role in improving the level of cholesterol and total lipid (Barillari et al., 2005), and the existence of vitamin C as demonstrated by Seyrek et al., (2004) reduce the concentration of triglyceride in the layer hens and the quail, Salah (2008) also confirmed that the addition of vitamin $\mathrm{C}$ resulted in a significant decrease in the concentration of triglycerides in male broiler breeders. These vitamins increase thyroid activity, Kuhn et al., (1993) reported that the thyroid gland is one of the most important glands in control of cholesterol and lipid metabolism.

Mashi (2017) also noted in a study conducted on male rabbits that the Aqueous extract of the Eruca sativa leaves ( $250 \mathrm{mg} / \mathrm{kg}$ orally for 30 days) causes a significant decrease $(\mathrm{P} \leq 0.05)$ in the triglyceride concentration, cholesterol, LDL-C, VLDL-C and elevation in HDL-C Compared with the control group, the hypolipidemic effect may be due to the enzyme 7- $\alpha$-Hydroxylase that activated by vitamin $C$ (a component of Eruca sativa leaves) that stimulates the conversion of serum cholesterol into bile acids, thus reducing serum cholesterol level as well as the ability of vitamin $\mathrm{C}$ to Inhibit HDL-C oxidation (Hillstrom et al., 2003), which was confirmed by AbdulRahman and Alkatan (2009) as they found a decrease in the concentration of triglycerides when they adds vitamin $\mathrm{C}$ to the laying hens.

El-Gengaihi et al., (2004) confirmed that Eruca sativa seeds oil improve the lipid profile after 4,8 and 12 weeks in rats $(0.5 \mathrm{~g}$ /day orally for 3 months $)$.The reduction of serum cholesterol and total lipid may be due to the unsaturated fatty acids (85\%) of Eruca sativa such as linoleic acid and linolenic acid (Thomas, 2002) or due to glucosinolate (a substance that inhibits lipid peroxidation) (Al-Doghachi et al., 2010).

The hypolipidemic effect of Eruca sativa may be due to some of its active components as saponins (Zamani et al., 2007) flavonoids, phenols, turbines and alkaloids (Asaduzzaman et al., 2010), as well as the glucosinolates (Wang et al., 1998).

Also, table (3) revealed that Eruca sativa treatment reduces the stress effects on both males and females quail at 42 days and 77 days age as represented in the reduction of AST and ALT values in treated groups, and the best effects were achieved when Eruca sativa treatment was continued for the whole period (7 - 77 days).

Results of the current study were in agreement with the study of Razooqi et al. (2014) in broiler, and with Al-Daraji and Razuki (2012) in layer breeders roosters, and with Razuki (2009) in laying chickens.

The reduction of AST and ALT values may return to activation of liver regeneration and functions due to its content of antioxidants as Kaempferol and quercetin and the glucosinolates (Jin et al., 2009 ; El-Fadaly et al., 2017), and also to its high content of sulphur, which activate the liver function and immune system 
Mesopotamia J. of Agric.

Vol. (47) No. (1) 2019
ISSN: 2224 - 9796 (Online)

ISSN: 1815 - $316 \mathrm{X}$ (Print)

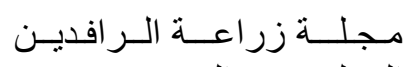

المجلد (47) العدد (1) 2019

(Alam et al., 2007). Also, Abdel-Rahman et al., (2015) showed that the Eruca sativa seed extract reduce lipid peroxidation and improve cellular antioxidants in rats.

In conclusion, the addition of crushed Eruca sativa seeds improve the lipid profile, and reduces stress condition and risk index in males and females quail, especially when given on the growth stage and before sexual maturity.

Table (3): Effect of adding* crushed Eruca sativa seeds to the ration on serum AST and ALT of males and females quail at age 42 and 77 days.

\begin{tabular}{|c|c|c|c|c|c|c|c|c|}
\hline \multirow[b]{3}{*}{ Treatments } & \multicolumn{4}{|c|}{ Male } & \multicolumn{4}{|c|}{ Female } \\
\hline & \multicolumn{2}{|c|}{$\begin{array}{l}\text { AST } \\
\mathrm{U} / \mathrm{L}\end{array}$} & \multicolumn{2}{|c|}{$\begin{array}{l}\text { ALT } \\
\mathrm{U} / \mathrm{L}\end{array}$} & \multicolumn{2}{|c|}{$\begin{array}{l}\text { AST } \\
\mathrm{U} / \mathrm{L}\end{array}$} & \multicolumn{2}{|c|}{$\begin{array}{l}\text { ALT } \\
\mathrm{U} / \mathrm{L}\end{array}$} \\
\hline & $\begin{array}{c}42 \\
\text { days }\end{array}$ & $\begin{array}{c}77 \\
\text { days }\end{array}$ & $\begin{array}{l}42 \\
\text { days }\end{array}$ & $\begin{array}{c}77 \\
\text { days } \\
\end{array}$ & $\begin{array}{c}42 \\
\text { days }\end{array}$ & $\begin{array}{c}77 \\
\text { days }\end{array}$ & $\begin{array}{c}42 \\
\text { days }\end{array}$ & $\begin{array}{c}77 \\
\text { days }\end{array}$ \\
\hline $\begin{array}{c}1^{\text {st }} / \text { Control } \\
\text { (Without } \\
\text { Eruca sativa seeds) }\end{array}$ & $\begin{array}{c}157.51 \\
\pm 8.15 \\
\text { A }\end{array}$ & $\begin{array}{c}243.92 \\
\pm 12.27 \\
\text { A }\end{array}$ & $\begin{array}{c}53.44 \\
\pm 1.47 \\
\mathrm{~A}\end{array}$ & $\begin{array}{c}17.35 \\
\pm 1.16 \\
\text { A }\end{array}$ & $\begin{array}{c}219.93 \\
\pm 15.99 \\
\text { A }\end{array}$ & $\begin{array}{c}217.12 \\
\pm 15.83 \\
\text { A }\end{array}$ & $\begin{array}{c}50.63 \\
\pm 2.55 \\
\mathrm{~A}\end{array}$ & $\begin{array}{c}82.48 \\
\pm 2.63 \\
\text { A }\end{array}$ \\
\hline $\begin{array}{l}2^{\text {nd }} / \text { Growth stage } \\
\text { (Eruca sativa seeds } \\
\text { from } 7-42 \text { days) }\end{array}$ & $\begin{array}{c}122.87 \\
\pm 3.27 \\
\text { B }\end{array}$ & $\begin{array}{c}232.08 \\
\pm 18.39 \\
\mathrm{AB}\end{array}$ & $\begin{array}{c}25.36 \\
\pm 2.75 \\
\mathrm{~A}\end{array}$ & $\begin{array}{c}15.92 \\
\pm 1.43 \\
\text { AB }\end{array}$ & $\begin{array}{c}184.31 \\
\pm 12.14 \\
\mathrm{AB}\end{array}$ & $\begin{array}{c}200.08 \\
\pm 14.17 \\
\mathrm{AB}\end{array}$ & $\begin{array}{c}23.92 \\
\pm 3.03 \\
\text { B }\end{array}$ & $\begin{array}{c}70.39 \\
\pm 2.30 \\
\mathrm{~B}\end{array}$ \\
\hline $\begin{array}{c}3^{\text {rd }} / \text { Production stage } \\
\text { (Eruca sativa seeds } \\
\text { from } 42-77 \text { days) }\end{array}$ & $\begin{array}{c}155.83 \\
\pm 7.28 \\
\text { A }\end{array}$ & $\begin{array}{c}193.27 \\
\pm 18.90 \\
\mathrm{AB}\end{array}$ & $\begin{array}{c}49.17 \\
\pm 1.79 \\
\mathrm{~A}\end{array}$ & $\begin{array}{c}14.79 \\
\pm 1.17 \\
\mathrm{AB}\end{array}$ & $\begin{array}{c}207.91 \\
\pm 20.07 \\
\text { A }\end{array}$ & $\begin{array}{c}173.42 \\
\pm 10.43 \\
\text { BC }\end{array}$ & $\begin{array}{c}48.60 \\
\pm 4.45 \\
\text { A }\end{array}$ & $\begin{array}{c}50.52 \\
\pm 3.38 \\
\text { C }\end{array}$ \\
\hline $\begin{array}{l}4^{\text {th }} / \text { Total stage } \\
\text { (Eruca sativa seeds } \\
\text { from } 7-77 \text { days) }\end{array}$ & $\begin{array}{c}119.16 \\
\pm 6.61 \\
B\end{array}$ & $\begin{array}{c}178.81 \\
\pm 21.89 \\
\text { B }\end{array}$ & $\begin{array}{c}24.41 \\
\pm 2.44 \\
\text { B }\end{array}$ & $\begin{array}{c}12.40 \\
\pm 1.76 \\
\mathrm{~B}\end{array}$ & $\begin{array}{c}147.21 \\
\pm 10.30 \\
\mathrm{~B}\end{array}$ & $\begin{array}{c}137.28 \\
\pm 11.11 \\
\mathrm{C}\end{array}$ & $\begin{array}{c}25.83 \\
\pm 2.48 \\
\text { B }\end{array}$ & $\begin{array}{c}40.47 \\
\pm 5.49 \\
\text { C }\end{array}$ \\
\hline
\end{tabular}

- Values are represent : Means \pm Standard Error.

- Different letters in each column mean significant differences at $(\mathrm{P} \leq 0.05)$.

-*Adding $6 \mathrm{~g} / \mathrm{kg}$ feed of crushed Eruca sativa seeds.

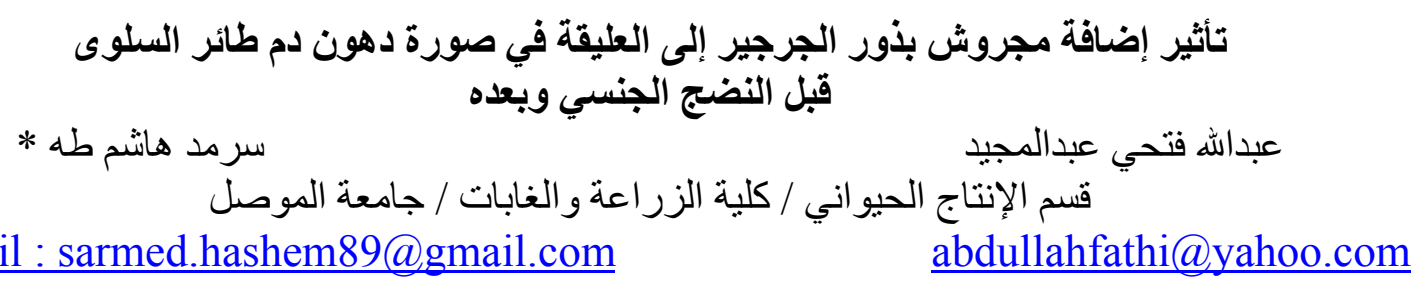

Email : sarmed.hashem89@gmail.com

\section{الخلاصـة}

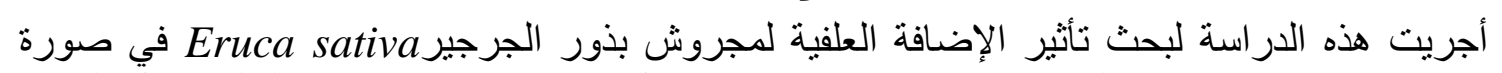

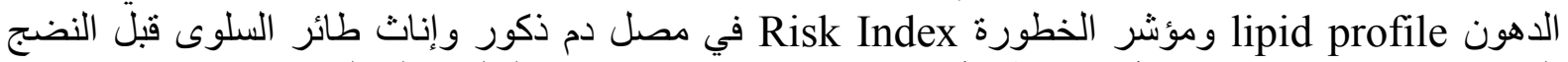

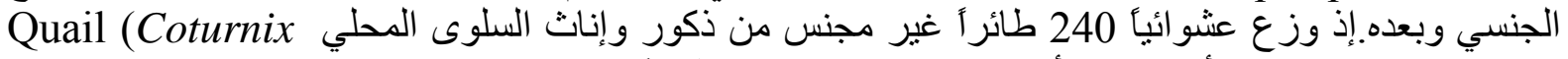
Coturnix)

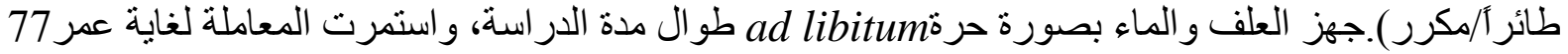
يو مأ، وكانت مجمو عات الدر اسة كالآتي: 


$$
\text { ا - المجموعة الأولى (مجموعة السبطرة):أعطبت الطيور عليقة أساسية بدون إضافات. }
$$

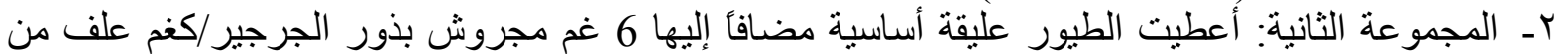
بداية التجربة (عمر 7 أيام)ولغاية عمر النضج الجنسي (عمر 42 يومأ)، ثم استبدلت بعليقة أساسية خالية من

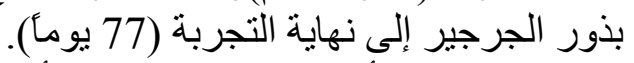

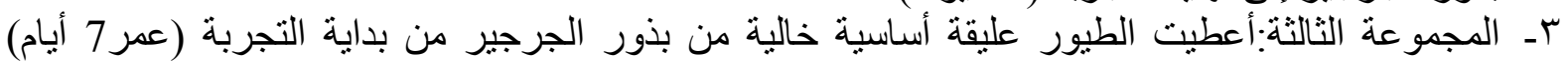

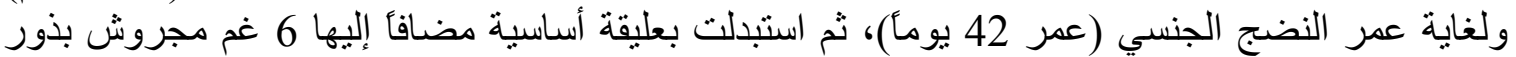
الجرجير/كغم علف إلى نهائة التجربة (الجنة (77 يوماً).

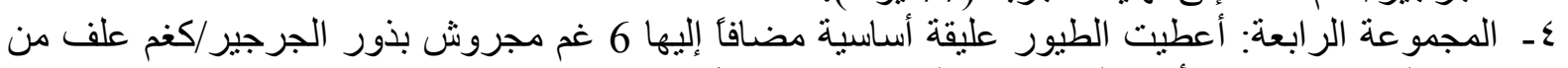

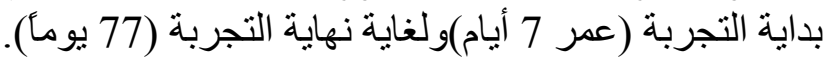

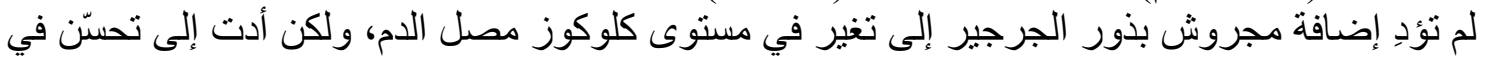

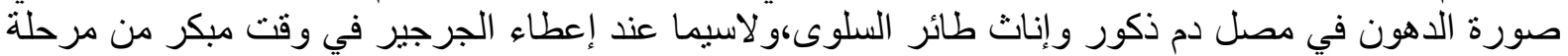

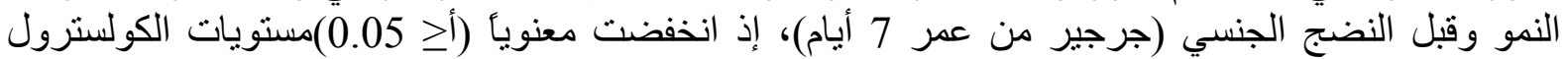
و الكليسيريدات الثنلاثية و VLDL-C مقارنة مع مجمو عة السيطرة.

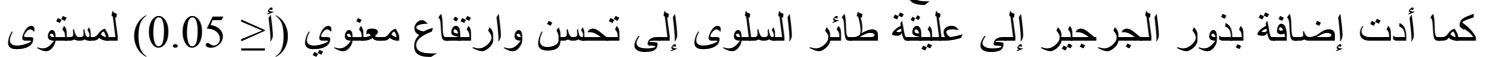

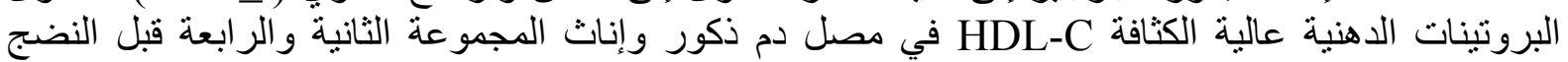

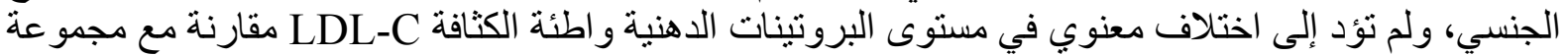

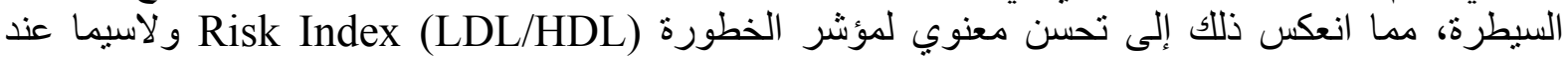
إضافة بذور الجرجير من عمر 7 أيام فانخفضت قِيمَهُ في الذكور والإناثن مقارنة مع مجموعة السبطرة عند

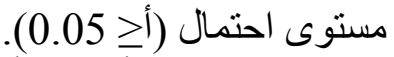
ومن ناحية أخرى، أن إضافة إنة مجروش بذور الجرجير أدت إلى تحسن في حالة الإجهاد لذكور و إناث طائر

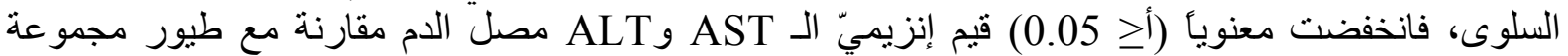
السيطرة.

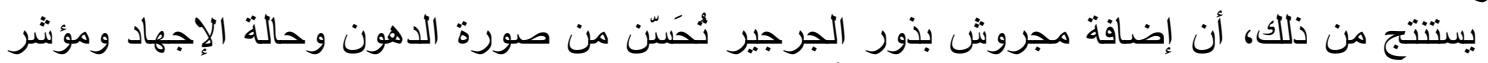
الخطورة عند ذكور و إناث طائر السلوى و لاسيما إذا أعطيت البذور في مرحلة النمو وقبل النضج الجنسي.

الكلمات المفتاحية: الجرجير ، البروتينات الدهنية واطئة الكثافة، البروتينات الدهنية عالية الكثافة، السمان.

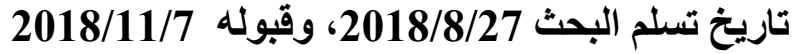

\section{References}

Abdel-Rahman, S. ; N. Shabana and A. Haggag (2015). Inhibition of NF-KB, bcl-2, and COX- 2 gene expression by Eruca sativa seeds extract in rat mammary gland carcinogenesis. Asian Pacific Journal of Cancer Prevention, 16 (18): 8411 8418.

Abdul-Jalil, T.Z. (2016). Phytochemicals Screening by GC/MS and Determination of Some Flavonol in Cultivated Iraqi Eruca sativa Dried Leaves Extract and its Biological Activity as Antioxidant. International Journal of Pharmacognosy and Phytochemical Research, 8(10): 1722-1730.

Abdul-Rahman, Saeb Younis and Montaha Mohmood AlKatan (2009). Effect of some antioxidants on some physiological and reproductive parameters in laying hens, Iraqi Journal of Veterinary Sciences. Fifth Scientific conference, Veterinary College. University of Mosul , 23 (2) - 377- 384.

Alam, M.S.; G. Kaur; Z. Jabbar; K. Javed, and M. Athar (2007). Eruca sativa Seeds Possess Antioxidant Activity and Exert A Protective Effect on Mercuric Chloride Induced Renal Toxicity. Food and Chemical Toxicology. 45(6):910920. 
Al-Dalaly, Basel Chamel (1994). Principles of Biochemistry. Dar of printing and publishing. ministry of higher education and scientific research. University of Mosul.

Al-Daraji, H.J. and R.H. Razuki.(2012). Effect of dietary supplementation with different levels of Eruca sativa seeds powder on blood plasma traits of White Hy-line laying breeder roosters. Kufa Journal for Agricultural Science, 1(4): 3142.

Al-Daraji, Hazim Jabar, Al-Hoiney Waleed Khalid Al-Hasany and Ali Sabah (2008). Birds blood physiology, Ministry of higher education and scientific research, Agriculture college. University of Baghdad.

Al-Doghachi, Essam Hussein, Sabah Neima Al-Thamir and Maher Al-Mohammad (2010). A clinical study of Antihyperlipidemic effects of Jamba oil (Eruca sativa Mil.) on serum blood. Kufa Journal for Agricultural Science. 2(1) 170-177.

Al-Eneezy, Mohanad Abed Alhasan Kareem (2004). Effect of pure extractions of Eruca sativa in the growth of some pathogenic bacteria. A Thesis. College of Sciences, Department of biotechnology, University of Baghdad.

Anonymous (1994). National Research Council (N. R. C.) Nutrient requirement of poultry $9^{\text {th }}$ revisited. National Academy Press, Washington, DC. UAS.

Anonymous (2000). statistical analysis system(SAS) / STAT User's Guide, Version 6.12. SAS. Inst. Inc., Cary, NC.

Ansari, M.N and Ganaie M.A. (2014). Ameliorative Effect of Rocket Leaves on Fertility in Streptozotocin-Induced Diabetic Rats. Int. Res. J. Biological Sci. 3(8):89-97.

Asaduzzaman, M. ; M.A. Afiya ; M.A. Islam ; R.I. Khan and A. Maruf (2010). Evaluation of the antidiabetic, antihyperlipidemic and hepatoprotective effect of Allium sativa L. in alloxan induced diabetic rats. Bangladesh Pharmaceutical Journal. 13(1):28-33.

Badee, A.Z.M. ; S.A. Hallabo and M.A.A. Aal (2003). Biological evaluation of Egyptian Eruca sativa seeds and leaves. Egyptian Journalof Food Science. 31 (1-2): 67-78.

Barillari, J. ; D. Cansitor ; F. Ferroni ; M. Paolini ; G.F. Pedulli and R. Iori (2005). Direct Antioxidant Activity of Purified Glucoerucin, in The Dietary Secondary Metabolite Continued in Rocket Eruca sativa Mill Seeds and Sprouts. Journal of Agricultural and Food Chemistry.53 (7): 2475- 2482.

Bell, L. and Wagstaff C. (2014). Glucosinolates, myrosinase hydrolysis products, and flavonols found in a rocket (Eruca sativa and Diplotaxis tenuifolia). Journal of Agricultural and Food Chemistry.62(20): 4481-4492.

Carr, M.E. ; J. Klotz, and M. Bergeron (2004). Coumadin resistance and the vitamin supplement "Noni". American journal of hematology, 77: 103-107.

Duncan, D.B. (1955) Multiple range and multiple F-test. Biometric. 11: 1-42.

El-Fadaly, H.A. ; S.M. El-Kadi ; M.M. El-Moghazy ; A.A. Soliman and M.S. ElHaysha (2017). Glucosinolates in Rabbit Nutrition: Biologically Detoxification, Growth Performance, Digestibility, Blood Constituents, and Carcass Characteristics. International Journal of Biological Sciences and Applications; 4(5): 43-55. 
El-Gengaihi, S.E., A.M. Salem, S.A. Bashandi, N.A. Ibrahim and S.R. Abd el-Hamid (2004). Hypolipidemic effect of some vegetable oils in rats. Food Agric\& Environment. 2 (2): 88 - 93.

Flanders, A., and S.M. Abdulkarim (1985). The composition of the seed oil of Tramira Eruca Sativa. Journal of the American Oil Chemists' Society. 62(7): 1134- 1135.

Hillstrom, R.J. ; A.K. Yacapin- Ammons and S.M. Lynch (2003). Vitamin C inhibits lipid peroxidation in human HDL. Journal of Nutrition; 133(10):3047-3051.

Jin, J. ; O.A. Koroleva ;T. Gibson ; J. Swanston ; J. Magan ; Y. Zhang ; I.R. Rowland and C. Wagstaff (2009). Analysis of phytochemical composition and chemoprotective capacity of rocket (Eruca sativa and Diplotaxis tenuifolia) leafy salad following cultivation in different environments. Journal of Agricultural and Food Chemistry, 57(12): 5227-5234.

Kuhn, E.R. ; L.R. Bergham ; L. Moons ; F. Vandesande ; E. Decuypere, and M. Darres. (1993). Hypothalamic and peripheral control of thyroid function during the life cycle of the chicken. In: Avian Endocrinology. Sharp P.J. (Ed.) Pp: 2946.

Mashi, S.K. (2017). Effect of Eruca sativa leaves extract on liver enzymes and lipid profile in phosphoric acid induced liver damage in male rabbits. Journal of Entomology and Zoology Studies. 5(6): 1011 -1015.

Mossa, J.S ; M.A. Al-Yahya, and I.A. Al-Meshal (1987). Medicinal Plants of Saudi Arabia. Vol. 1. King Saud Univ. Libraries Publications. Riyadh, KSA.

Pignone, D. and M.A. Ngu (1995). Collection and conservation of rocket genetic resources: The Italian contribution. International Plant Genetic Resources, Italy. Pp:8- 11.

Razooqi, Raad Hatem ; Hayder kadhim Shkeer ; Yousif Okaab Alwan and Marwan Ibrahim Hayder (2014). Effect of Eruca sativa oil (ESO) on broiler performance and some blood traits. International journal of advanced biological research, 4 (4): 479-482.

Razuki, Raad Hatem (2009). Effect of rocket salad (Eruca sativa Mill) seeds on productive and reproductive traits of males and females of laying chickens. A dissertation, Agriculture College, University of Baghdad.

Salah, Sinan issam Al-Deen (2008). Effect of using Vitamin C, A and Fenugreek seeds on Some Physiological and Histological Parameters of Male Arber Acers Breeders. A Thesis. College of Agriculture and Forestry. University of Mosul.

Seyrek, K. ; C. Yenisey ; M. Serter ; F. Karginkiral ; P.A. Ulutas and H.E. Bardakcioglu (2004). Effects of dietary vitamin C supplementation on some serum biochemical parameters of laying Japanese Quails exposed to heat stress $\left(34.8^{\circ} \mathrm{C}\right)$. Review Med. Vet., 155(6): 339-342.

Steel, R.G.D. and J.H. Torrie (1960). Principles and Procedures of Statistics. McGraw- Hill Book. Co., Inc., New York, N. Y. Pp 481.

Thomas, A. (2002). Fats and fatty oils. Ullmanns Encyclopedia of Industrial Chemistry. Weinheim. Wiley-VCH. 
Uğur, A. ; I. Süntar ; S. Aslan ; I.E. Orhan ; M. Kartal ; N. Şekeroğlu ; D. Eşiyok and B. Şener (2010). Variations in fatty acid compositions of the seed oil of Eruca sativa Mill. caused by different sowing periods and nitrogen forms. Pharmacognosy Magazine. 6(24):305-308.

Wang, L.F. ; H. Luo ; M. Miyoshi ; T. Imoto ; Y. Hiji and T. Sasaki (1998). Inhibitory effect of Gymnemic acid on intestinal absorption of oleic acid in rats. Canadian Journal of Physiology and Pharmacology. 76(10-11): 1017-1023.

Zamani, M. ; O.A. Rahimil ; R. Mahdavi ; M. Nikbakhsh ; V.M. Jabbari1 ; H. Rezazadeh ; A. Delazar ; L. Nahar and D.S. Sarker (2007). Assessment of the anti-hyperlipidemic effect of Citrullus colocynthis. Brazilian. Journal of Pharmacognosy. 17 (4): 492-496. 